\title{
Stress Analysis of Cylindrical Steel Storage Liquid Tanks During the Instantaneous Loads
}

\author{
${ }^{1}$ Ali İhsan Çelik, ${ }^{2}$ Tahir Akgül \\ ${ }^{1}$ Adıyaman University, Besni Vocational High School \\ Turkey \\ acelik@adiyaman.edu.tr \\ ${ }^{2}$ Sakarya University Faculty of Technology, Civil Engineering \\ Turkey
}

\begin{abstract}
Cylindrical steel tanks are widely used to store variety liquids such as water, petroleum, and industrial chemicals. They also have been becoming widespread for cooling in nuclear power plants last years. Steel liquid tanks can be exposed during earthquakes and also they can cause great financial and environmental damage with their containing petroleum or other hazardous chemical liquid. Explicit Dynamic tool in ANSYS Workbench reveals accurate results in dynamic analysis of structures under instantaneous and short forces. The Explicit Dynamic tool uses Eulerian Body with water to provide interaction between the water and the shell. El-Centro earthquake 0,22 seconds data was used as displacement force for to see the shear stress on the tank. The tank was covered with epoxy carbon material using the Workbench ACP tool to reduce shear stress.
\end{abstract}

Keywords: Stress Analysis, Eulerian Body Mesh, Epoxy Carbon.

\section{Introduction}

This paper is included two main sections. In the first section was carried out the explicit dynamic analysis and from the second section, tank wall was strengthened with epoxy carbon which is a composite material. If product needs to survive impacts or short-duration high-pressure loadings, it can be improved its design with ANSYS explicit dynamics. Specialized problems require advanced analysis tools to accurately predict the effect of design considerations on product or process behavior. Gaining insight into such complex reality is especially important when it is too expensive or impossible to perform physical testing. The ANSYS explicit dynamics suite enables to capture the physics of short-duration events for products that undergo highly nonlinear, transient dynamic forces. Specialized, accurate and easy-to-use tools have been designed to maximize user productivity. With ANSYS, it can be obtained insight into how a structure responds when subjected to severe loadings. Algorithms based on first principles accurately predict complex responses, such as large material deformations and failure, interactions between bodies, and fluids with rapidly changing surfaces.

There are some studies specific about this topic. Akira M. and Katsuhisa F. proposed a nonlinear dynamic analysis method concerning the coupled vibration between fluid and structure using an explicit method. The proposed method uses a shell element which can take into account the geometric nonlinearity characteristics and a solid element which follows Euler's equation. The ALE method is applied to the coupling analysis between fluid and structure, and the explicit time integration method is used for the time-history response analysis [1]. Vaibhav Mittal et al, investigated the maximum hoop stress and shear stress in the tank wall under the blast using coupled euler - langrange formulation. They observed that stresses and liquid sloshing heights in the tank increase with decreasing scaled distance of the explosive material and increasing aspect ratio, i.e. height to radius ratio [2]. In the figure 1 and 2 are seen deformation of tanks due to sloshing subjected the earthquake load. 


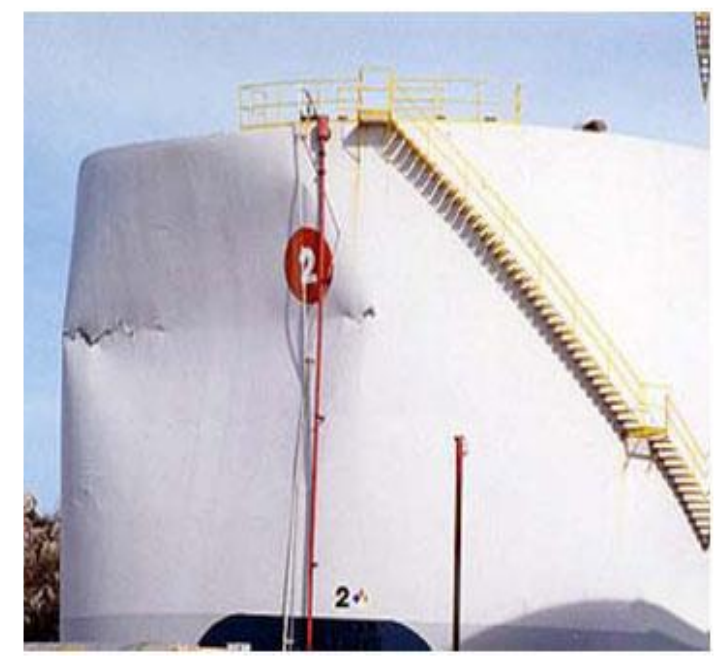

Fig. 1: Sloshing damage to upper shell of tank.

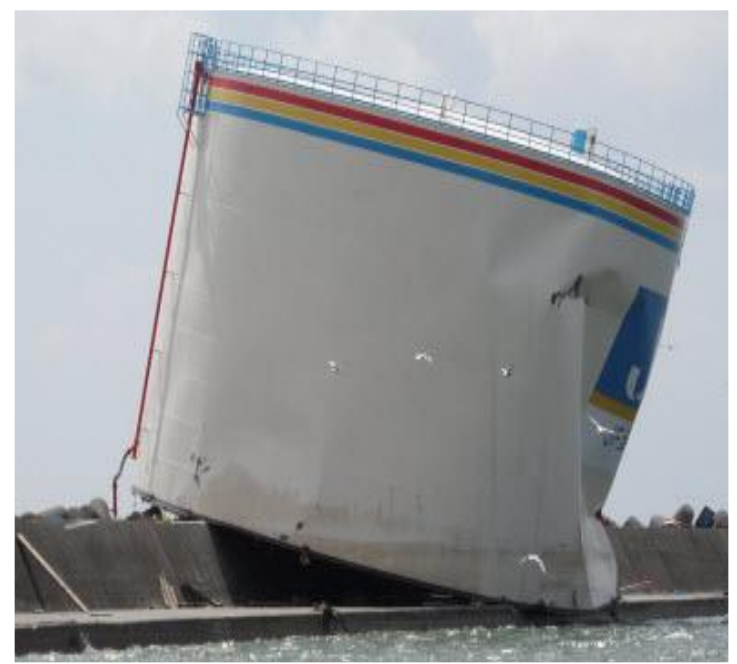

Fig. 2: Deformation Tank Sliding.

In the ANSYS Workbench, defaults are safe and reasonable values for most options, which means that spend less time setting up and running problems and more time optimizing products for performance, durability and cost, as well as removing design flaws [3].

Seddon CM et al, performed Preliminary analysis of Fuel Tank Impact, they developed numerical models using LS DYNA explicit dynamics software. This particular software was selected due to the use of explicit formulation within the program, which is particularly suitable for problems with short durations such as impact or explosion. [4].

Fluid-structure interaction problems are extremely complex; therefore, analytical solution methods are limited to understanding seismic behavior. Capabilities of finite element analysis provide deeper understanding asses seismic behavior cylindrical steel tanks. There are many explicit dynamic analyses about the exposed pressure and exploded tanks. However, there is no explicit dynamic analysis using eulerian body mesh method for cylindrical steel tanks which are exposed to hydrodynamic pressures under instantaneous earthquake loads. In this study is concentrated on the shear stress analysis of cylindrical steel tanks subjected instantaneous short duration earthquake loading and strengthening with epoxy carbon is recommended for exciting tanks. 


\section{Explicit Dynamics Analysis}

Variety of engineering simulations can be performed with the Workbench Explicit Dynamic method. ANSYS explicit dynamics a transient explicit dynamic, which includes of nonlinear dynamic behavior of solid, fluid, gases and their interaction. A typical simulation consists of setting up the model, interactions and the applied loads, solving the model's nonlinear dynamic response over time for the loads and interactions, then examining the details of the response with a variety of available tools.

The Explicit Dynamics application has objects arranged in a tree structure that guide through the different steps of a simulation. By expanding the objects, it is exposed the details associated with the object, and it can be used the corresponding tools and specification tables to perform that part of the simulation. Objects are used, for example, to define environmental conditions such as contact surfaces and loadings, and to define the types of results are wanted to have available for review.

An Explicit Dynamics analysis is used to determine the dynamic response of a structure due to stress wave propagation, impact or rapidly changing time-dependent loads. Momentum exchange between moving bodies and inertial effects are usually important aspects of the type of analysis being conducted. This type of analysis can also be used to model mechanical phenomena that are highly nonlinear. Nonlinearities may stem from the materials, (for example, hyperelasticity, plastic flows, failure), from contact (for example, high speed collisions and impact) and from the geometric deformation (for example, buckling and collapse). Events with time scales of less than 1 second (usually of order 1 millisecond) are efficiently simulated with this type of analysis.

\subsection{Eulerian (Virtual) Body Solver}

In an Eulerian reference frame, the grid remains stationary throughout the simulation. Material flows through the mesh. The mesh does not therefore suffer from distortion problems and large deformations of the material can be represented. Because of large deformations of steel tank material, using an Eulerian reference frame is preferable.

Solid, Liquid and Gaseous materials can be used with an Eulerian (Virtual) reference frame in the Explicit Dynamics system. Because of the computational cost and approximate tracking of material interfaces, the Eulerian reference frame should be used only when very large deformation or flow of the material is expected.

A volume of fluid (VOF) method is used track the amount of material in each cell. Each material has a volume fraction and the sum of the volume fraction of each material, plus the volume fraction of void, will equate to unity.

$$
\sum_{i=1}^{i=n m a t} F_{i}+F_{\text {void }}=1
$$

Nearly all isotropic material properties can be used in an Eulerian reference frame to represent solids, liquids or gases. Special treatment is required to allow calculation of the strain rates, pressure and stresses in each material in a cell, and also to calculate a resultant stress tensor which is then used to calculate cell face impulses, momentum and mass transport [3].

\subsection{Explicit Fluid Structure Interaction}

In the Explicit Dynamics system, solid bodies can be assigned either a Lagrangian reference frame or an Eulerian (Virtual) reference frame. The reference frames can be combined in the simulation to allow the best solution technique to be applied to each type of material being modelled. During the simulation, bodies represented in the two reference frames will automatically interact with each other. For example, if one body is filled with steel using a Lagrangian reference frame, and another body filled with water using an Eulerian reference frame, the two bodies will automatically interact with each other if they come into contact. The interaction between Eulerian and Lagrangian bodies provides a capability for tightly coupled two-way fluid structure interaction in the Explicit Dynamics system.

In the simple example below, a body with Lagrangian reference frame (grey) is moving from left to right over a body with Eulerian reference frame. As the body moves, it acts as a moving boundary in the Euler domain by progressively covering volumes and faces in the Euler cells. This induces flow of material in the Euler Domain. At the same time, a stress field will develop in the Euler domain which results in external forces being applied on the moving Lagrangian body. These forces will feedback into the motion and deformation (and stress) of the Lagrangian body. 


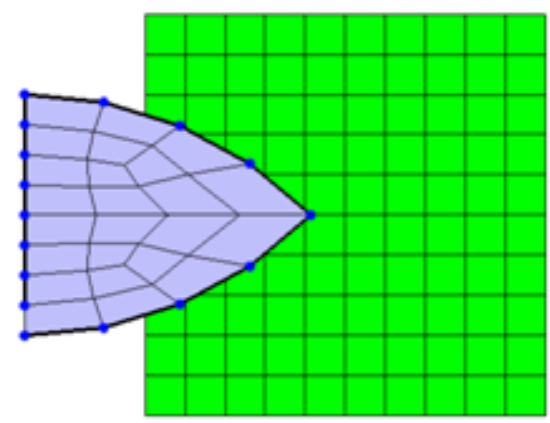

Fig. 3: Eulerian reference frame [3].

In more detail, the Lagrangian body covers regions of the euler domain. The intersection between the Lagrangian and Eulerian bodies results in an updated control volume on which the conservation equation of mass, momentum and energy are solved.

At the same time, the normal stress in the intersected Euler cell will act on the intersected area of the Lagrangian surface.

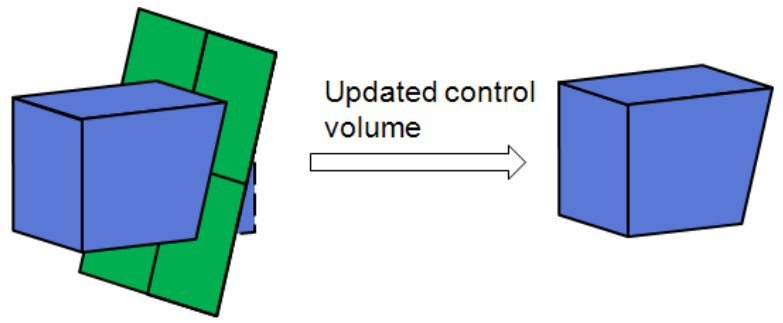

Fig. 4: Eulerian reference frame [3].

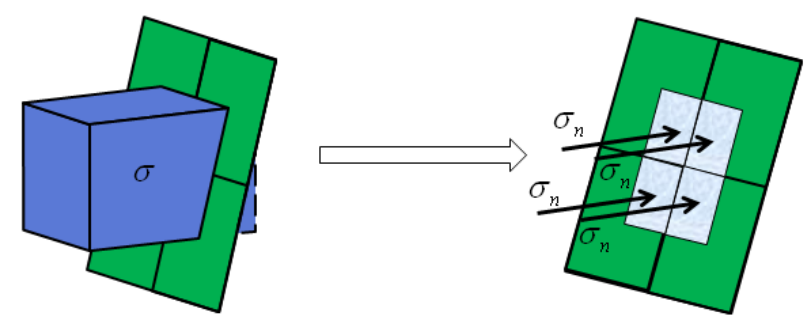

Fig. 5: Normal stress in the intersected Euler cell [3].

This provides a two-way closely coupled fluid-structure (or more generally Eulerian-Lagrangian) interaction. During a simulation, the Lagrangian structure can move and deform. Large deformations may also result in erosion of the elements from the Lagrangian body. The coupling interfaces are automatically updated in such cases.

For accurate results when coupling Lagrangian and Eulerian bodies in Explicit Dynamics it is necessary to ensure that the size of the cells of the Euler domain are smaller than the minimum distance across the thickness of the Lagrangian bodies. If this is not the case, it may be seen leakage of material in the Euler domain through the Lagrange structure [5].

\section{Approbation of Numerical Simulation Technique}

Explicit Dynamic application has objects arranged in a tree structure that guides a simulation through different steps. The objects are expanded to reveal the details associated with the object, and related tools and specification sheets can be used to perform this part of the simulation. Objects are used to describe environmental conditions, such as contact surfaces and loads, and to describe the types of results desired to be presented for viewing. 
The numerical simulations of the model tank and the water contents are shown in Table 1 . These values have been determined by the American Petroleum Institute (API 650) taking into account the standards for steel cylindrical water tanks.

Table 1: Parameters of Model Tank.

\begin{tabular}{|l|c|}
\hline Parameter and unit & Values \\
\hline Inner diameter of tank m. & 15,08 \\
\hline Tank height without roof $\mathrm{m}$. & 11,31 \\
\hline Tank height with roof $\mathrm{m}$. & 11,31 \\
\hline Water height $\mathrm{m}$. & 10 \\
\hline Shell thickness $\mathrm{m}$. & 0,012 \\
\hline Bottom plate thickness m. & 0,012 \\
\hline Density of tank steel $\mathrm{kg} / \mathrm{m}^{3}$ & 7850 \\
\hline Density of water $\mathrm{kg} / \mathrm{m}^{3}$ & 1000 \\
\hline Young's modulus of tank wall Gp. & 200 \\
\hline Poisson's ratio of tank wall $\boldsymbol{v}$. & 0,3 \\
\hline Bulk modulus of elasticity of water Gp. & 2.2 \\
\hline
\end{tabular}

The dimensions of the cylindrical water tank and the water in it are shown in figure 6.

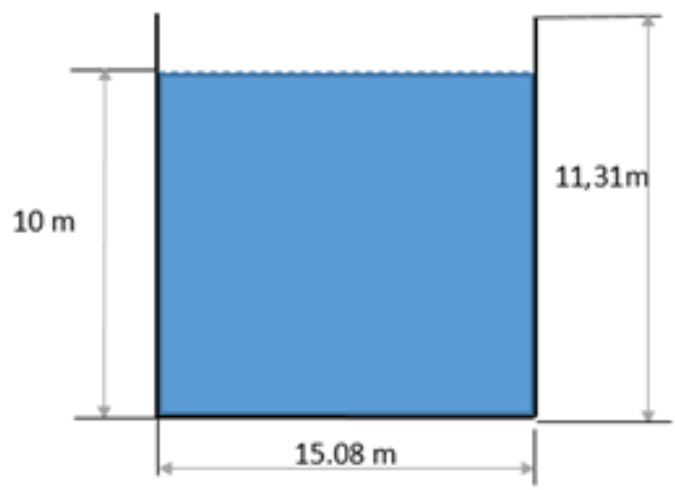

Fig. 6: Dimension of cylindrical steel tank.

An Explicit Dynamics analysis is used to determine the dynamic response of a structure due to stress wave propagation, impact or rapidly changing time-dependent loads. Momentum exchange between moving bodies and inertial effects are usually important aspects of the type of analysis being conducted. This type of analysis can also be used to model mechanical phenomena that are highly nonlinear. Nonlinearities may stem from the materials, (for example, hyperelasticity, plastic flows, failure), from contact (for example, high speed collisions and impact) and from the geometric deformation (for example, buckling and collapse). Events with time scales of less than 1 second (usually of order 1 millisecond) are efficiently simulated with this type of analysis[3]. Because of analysis explicit dynamic, El-Centro earthquake values of 022 seconds were used as displacement force.

\section{Performed Explicit Dynamic Analysis and Evaluation of Total Deformation}

After all adjustments were made, dynamic analysis was started and the deformation results of open top tank model are demonstrated in figure 7. The image was given in the form of a whole and half geometry for the understanding effect sloshing of the water. It is observed that, total deformation is more on the upper side of steel tank due to sloshing effect of water. Minimum deformation is $0,129 \mathrm{~m}$. and maximum deformation is $1,168 \mathrm{~m}$. 


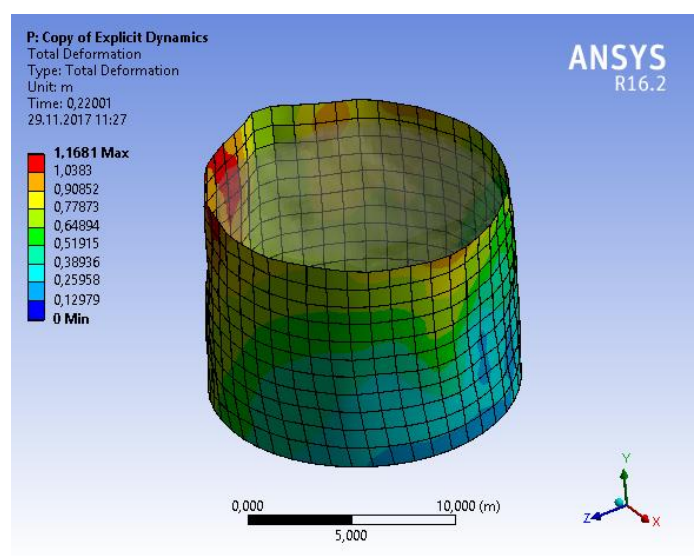

a) Total Deformation

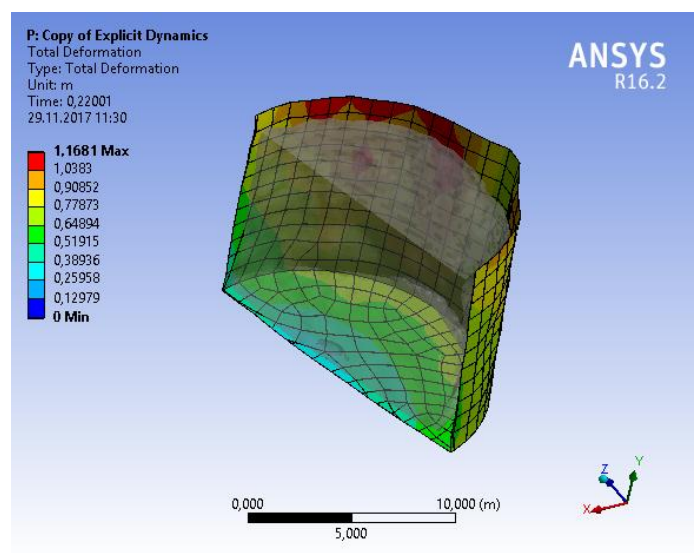

b) Half Geometry of Total Deformation

Fig. 7: Total Deformation of Open Top Tank.

\subsection{Strengthening of Cylindrical Steel Tank}

One of the considerable aims of this article is to strengthen the tank exposed to deformation under the loads of the earthquake. The performance of cylindrical steel tanks under extreme loading conditions, especially for blast and impact conditions, is of great importance despite the low probability for such events to occur. Due to catastrophic consequences of structural failure, it is crucial to improve the resistance of cylindrical tanks against the impact of deformation. It is possible to replace the steel tank with the new undeformable a tank made of thicker shell, but this will increase the cost considerably. Strengthening of existing tank at low cost is great importance.

Composite materials are widely used to fabricate cylindrical shells which can be the structural elements in storage tanks, cisterns for transportation pressure vessels, rocket components. The theory of cylindrical shells and its application to the analysis of cylindrical structures is described in numerous publications[6]. Stress-Strain graphics of composite materials is given in Figure 8.

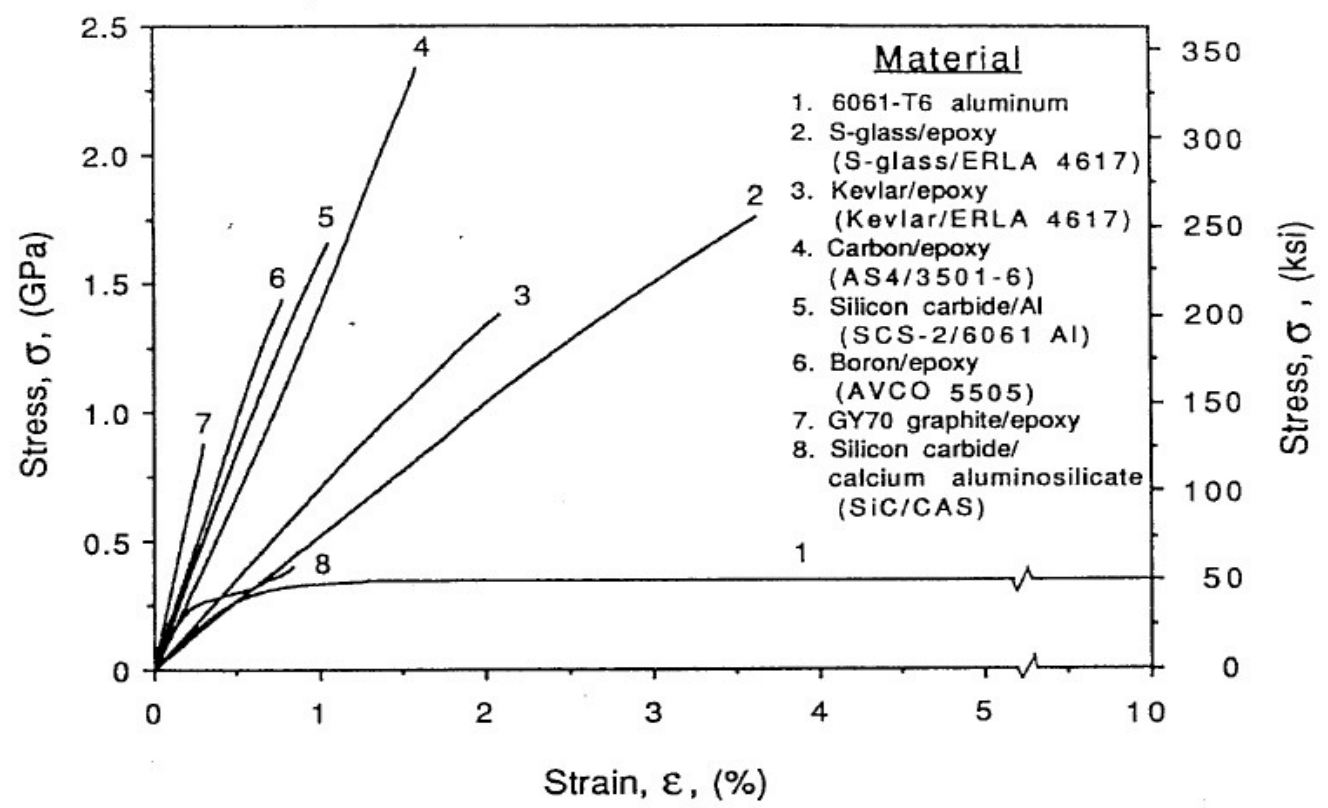

Fig. 8: Stress-Strain Graph of Some Composite Materials [7].

Composite material represents the fusion or combination of two different and insoluble materials. These materials remain distinguishable at the microscopic scale. Although each individual phase has its own properties, the properties and 
structural durability of the composite material is superior to each acting on its own [8]. The materials consist of a fibrous reinforcements bonded together with a matrix material. Allow the stiffness and strength of the material to change with direction of loading.

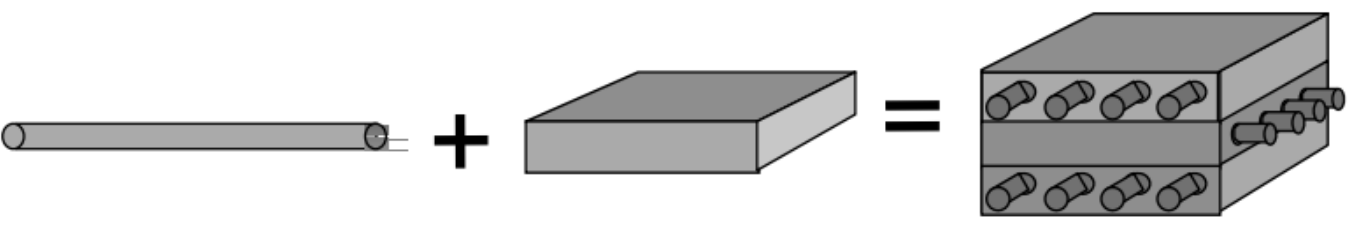

Fig. 9: Composition of Composites Materials [7].

Fiber/Filament

Reinforcement

- High strength

- High stiffness

- Low density
Matrix

- Good shear properties

- Low density

- Good shear properties

\section{Composite}

- High strength

-High stiffness

- Low density

Composite materials are widely used in aerospace structures and pressure vessel tanks, especially to protect against high air pressure.
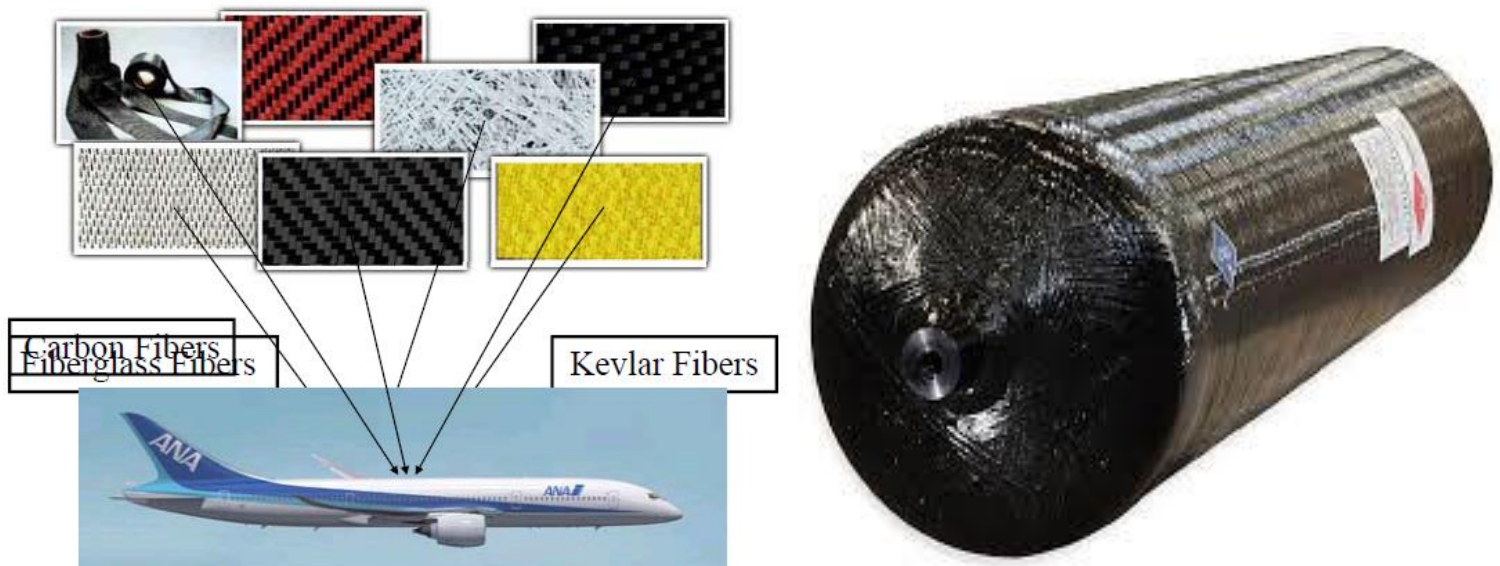

Fig. 10: Using Area of Composites Materials $[9,10]$.

The ANSYS Composite Prepost (ACP) module was used to perform the structural analysis of the composite wrap around the cylindrical water tank and the analyses were carried out. ANSYS is able to model and examine composite structures in more detail in this module.

Composite modeling in Workbench provides the ability to account for variability in the mechanical properties of composite materials due to any scalar user-defined quantity. Temperature, Shear Angle (defined through ACP-Pre draping), and Degradation Factor are predefined variables in Workbench Engineering Data to be used for refining a composite material's behavior.

-When plies of fabric are used in a laminate. The angle of the fabric warp is used as the ply direction angle. The fabric angle is enclosed in parentheses to identify the ply as a fabric ply.

- When the laminate is composed of both fabric and tape plies (a hybrid laminate). The parentheses around the fabric plies will distinguish the fabric plies from the tape plies.

- When the laminate is symmetrical and has an odd number of plies, the center ply is overlined to indicate that it is the midplane. 


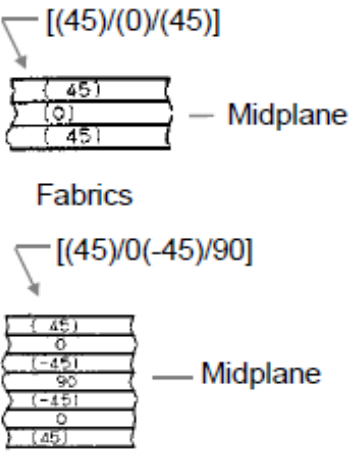

Tapes \& Fabrics

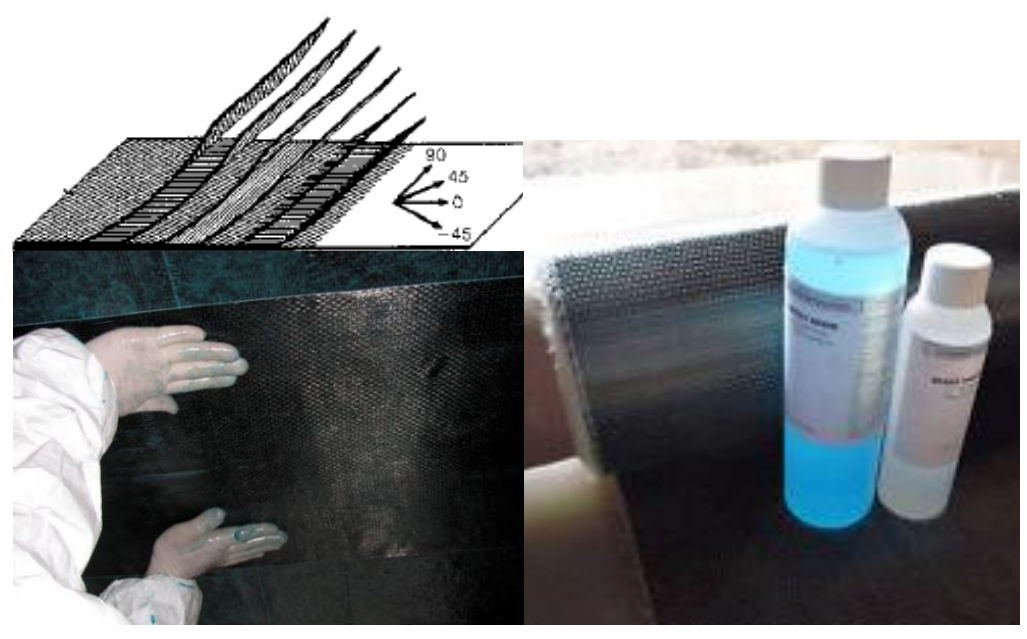

Fig. 11: Using Epoxy Carbon.

These specified angles are the helical of the fibers that it is spirally wrapped. In the program, fiber angles can be changed parametrically. The adjustment of the ideal angle of the layers is effective in strengthening. Determining of angles and tan tank are shown in figure $12[9,11]$.
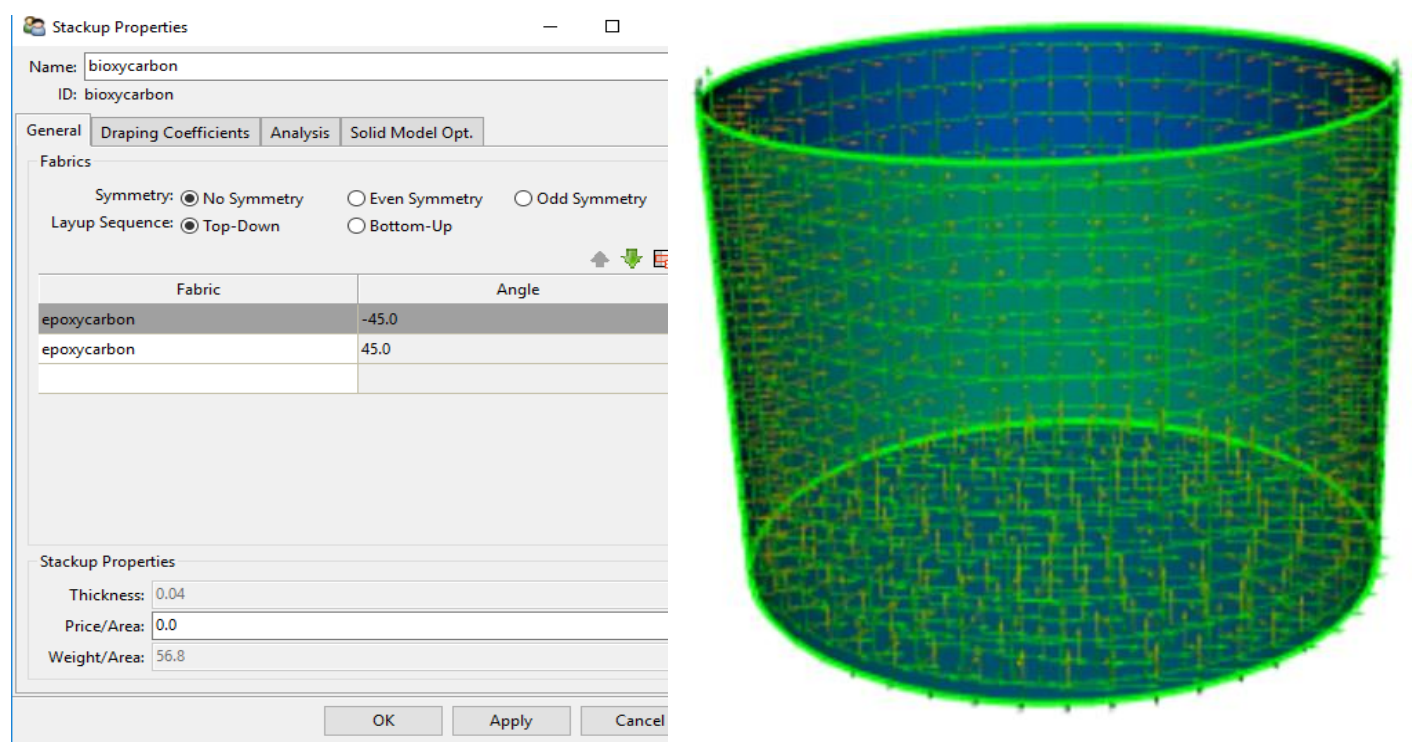

Fig. 12: Application of Epoxy Carbon in ACP.

\section{Results and Discussion}

In this section, it will be evaluated the total deformation results of open and shear stress after strengthening on wall with epoxy carbon.

\subsection{Result of Total Deformation}

After the water tank was strengthened with epoxy carbon, the deformation shape obtained is shown in figure 13. This figure when compared with figure 7 , it is seemed that deformation is decreased particularly caused due to sloshing on the upper side of tank. 


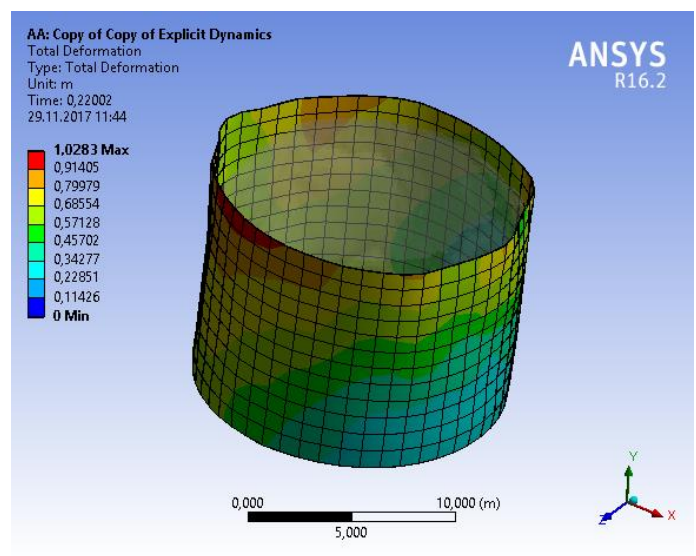

a) Total Deformation

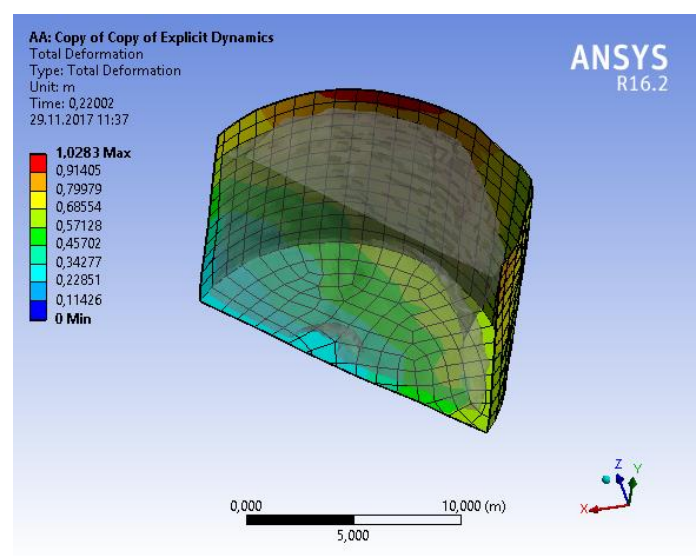

b) Total Deformation Half Section

Fig. 13: Strengthened water tank deformation.

From figure 14 it is seen that deformation in graph total deformation values decreased after strengthened water tan with epoxy carbon. After strengthening water tank, the maximum deformation value decreased to $1.08 \mathrm{~m}$, while it remained at $1.28 \mathrm{~m}$.

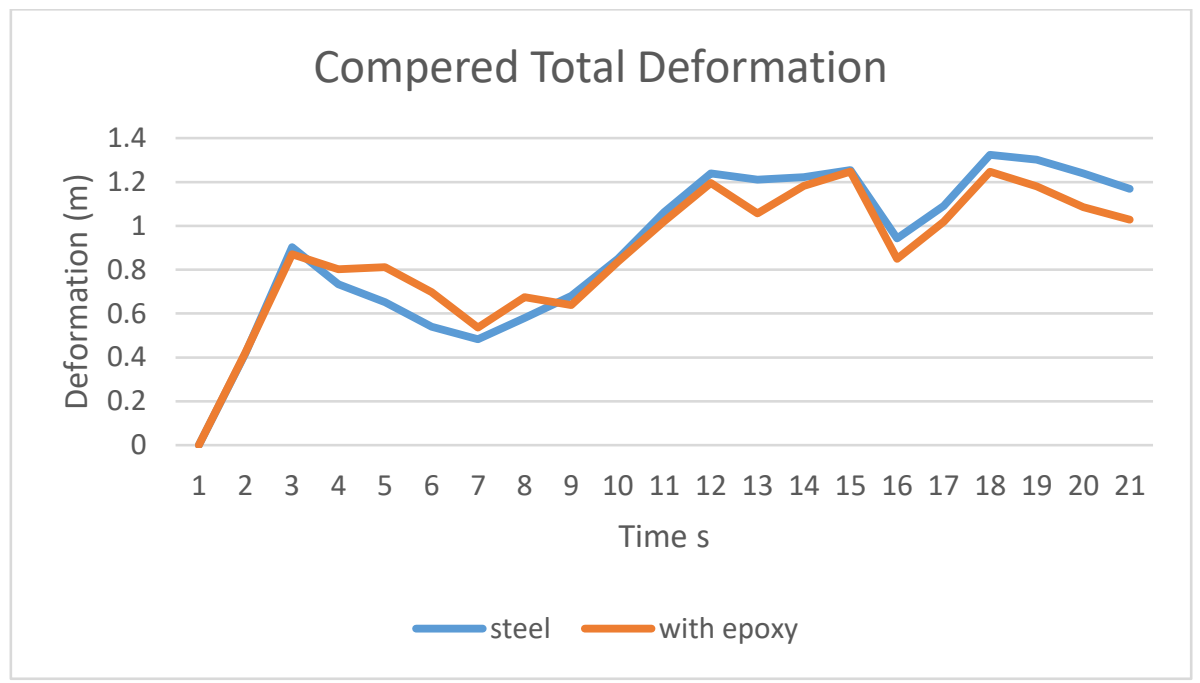

Fig. 14: Comparison of total deformation.

\subsection{Result of Shear Stress}

One of the finest results obtained in this study is shear stress. It is obvious that maximum shear stress value was higher than allowable stress (API 650) on the without strengthening tank, the shear stress value decreased this value after strengthening of tank with epoxy carbon. 


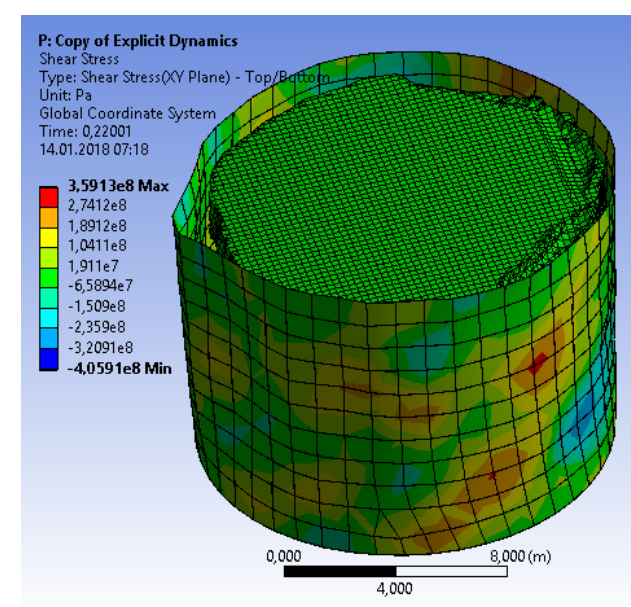

a) Shear Stress
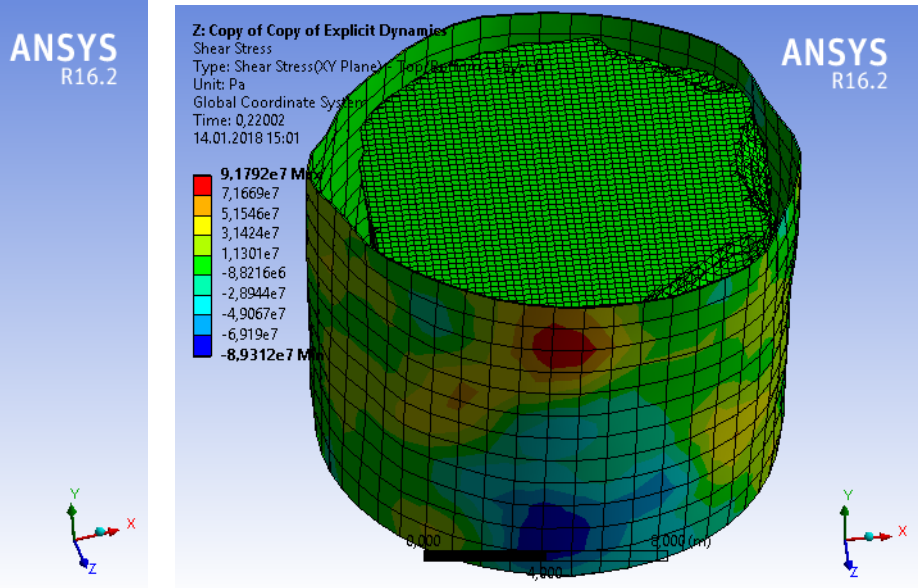

b) Shear Stress after apply epoxy

Fig. 15: Sher Stress.

Initially, shear stress was $359 \mathrm{Mpa}$. This value higher than $202 \mathrm{Mpa}$. (available API 650). After strengthening of tank shear stress decreased to $91,79 \mathrm{Mpa}$. While shear stress is higher in the bottom of the a) tank, the b) tank is scattered over the tops due to strengthening. Figure 16 shows maximum shear stress before strengthening and after strengthening.

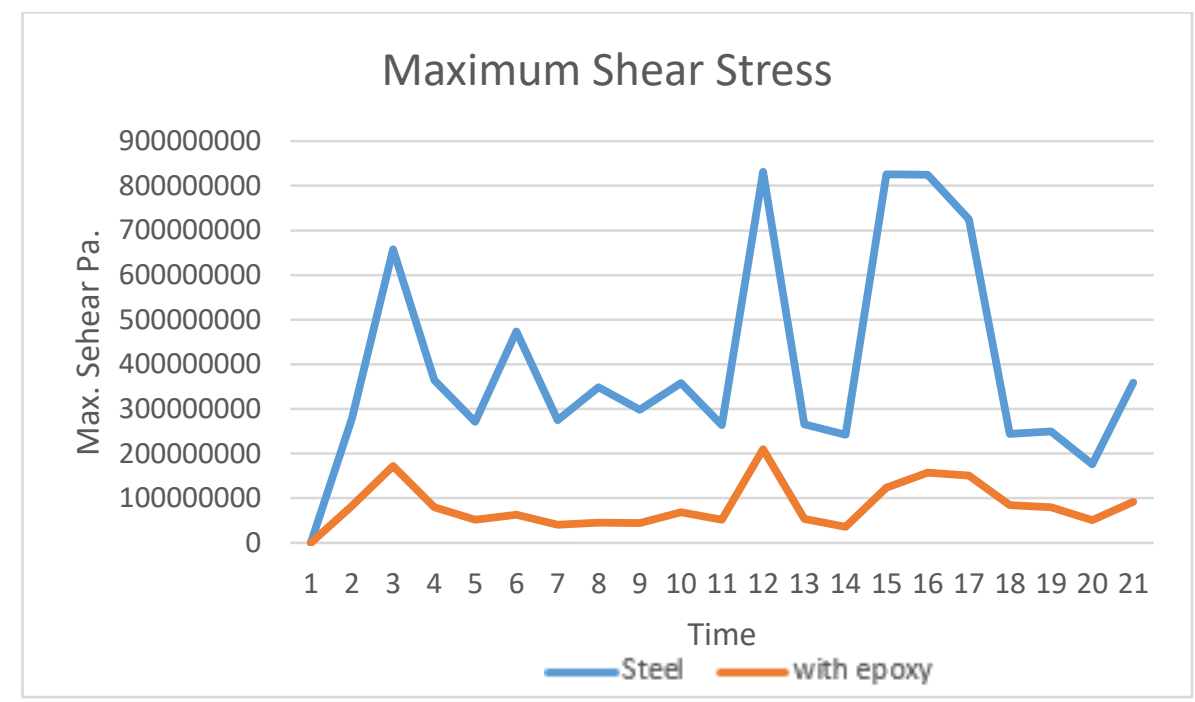

Fig. 16: Maximum Shear Stress Graph.

\section{Conclusion}

Explicit Dynamic analysis was performed to see the plastic deformation on the tank. Euler body mesh provides a good interaction between water and shell. Maximum deformation occurred upper side of tank. the effect of 0.22 seconds an earthquake load for explicit dynamic impact analysis was clearly observed. Capabilities of Lagrangian and Eulerian bodies in Explicit Dynamics analysis is showed that shear stress of cylindrical steel storage tanks can be investigated subjected short duration earthquake loads.

Moreover, epoxy carbon has serious strengthening effect on the tank's shell. As a result, it can be said, existing cylindrical steel water tanks can be protected with epoxy carbon from under the instantaneous seismic load. 


\section{References}

[1] A. Maekawa and K. Fujita, "Explicit Nonlinear Dynamic Analysis of Cylindrical Water Storage Tanks Concerning Coupled Vibration Between Fluid and Structure," ASME 2008 Pressure Vessels and Piping Conference, vol. 8, Seismic Engineering Chicago, Illinois, USA, 2008.

[2] V. Mittal, T. Chakraborty, V. Matsagar, "Dynamic analysis of liquid storage tank under blast using coupled EulerLagrange formulation," Thin-Walled Structures, vol. 84, pp. 91-111, 2013, 2014.

[3] ANSYS 16.2, Help Document.

[4] C. M. Seddon, K. Moodie, A. M. Thyer, and Moatamedi, "Preliminary analysis of fuel tank impact," The University of Salford, UK, 2004. [Online]. Available: https://usir.salford.ac.uk/318/1/Moatamedi_1.pdf

[5] Explicit fluid structure interaction, [Online]. Available: https://www.sharcnet.ca/Software/Ansys/17.0/enus/help/exd_ag/exp_dyn_theory_expl_flui_struct_582.html

[6] V. V. Vasiliev, E. V. Morozov, "Composite materials and structure materials," Elsevier 1td., no. 685.

[7] I. M. Daniel, O. Ishai, "Engineering Mechanics of Composite Materials," Oxford University Press, New York, 1994.

[8] Distriguish between composites and metal alloys. [Online]. Available: https://quizlet.com/110694842/ch-9flash-cards/

[9] S. Douglas Cairns, A. Lysle,"Composite Materials for Aircraft Structures," Department of Mechanical and Industrial Engineering Montana State University, ME 463 Composites, 2009, [Online]. Available: http://www.montana.edu/dcairns/documents/composites/MSUComposites2009.pdf

[10] Type 3 Cng Tanks, [Online]. Available: http://wercng.com/cng-tanks/type-3-cng-tank-type-3-cng-cylinder/

[11] Carbon Fibre Structural Reinforcement Products and Applicationscontent, [Online]. Available: http://gemite.com/wp-content/uploads/2013/04/Carbon_Structural_Strengthening.pdf 